\title{
Chitosan decorated copper nanoparticles as efficient catalyst for one-pot multicomponent green synthesis of novel quinoline derivatives
}

\author{
K.S. Alghamdi ${ }^{1}$, N.S.I. Ahmed ${ }^{1,2}$, D. Bakhotmah ${ }^{1}$, M. Mokhtar ${ }^{1,3^{*}}$ \\ 1. Chemistry Dept., Faculty of Science, King Abdulaziz University, P.O. Box 80203, Jeddah 21589, Saudi Arabia \\ 2. Medicinal Chemistry Dept., National Research Centre, El Buhouth St., Dokki, Cairo 12622, Egypt \\ 3. Physical Chemistry Dept., National Research Centre, El Buhouth St., Dokki, Cairo 12622, Egypt
}

\begin{abstract}
Chitosan decorated copper nanoparticles catalysts (CSCuNPs) were synthesized via reduction methods utilizing green protocol. The catalytic performance of CSCuNPs were tested for one-pot multicomponent reaction (MCR) using four reactant components: aromatic aldehydes, dimedone, ammonium acetate and ethylcyanoacetate under ultrasonic irradiation. The best catalyst (Cu-CS-NPs) that provided good conversion reaction yield and high turnover frequency (TOF) utilizing a facile and fast ultrasonic process was characterized using FTIR, TGA, XRD, TEM and XPS techniques. Generalization of the scope of the proposed catalytic process was studied using different aldehydes and excellent products yields and high TOF in even shorter reaction time (5 min.) was attained. Recyclability performance of the catalyst over five times re-use without detectable loss in product yield was recorded. The current method is green process utilizing environmentally benign catalyst and considered to be promising sustainable protocol for the synthesis of fine chemicals.
\end{abstract}

Keywords: Chitosan-copper NPs; Quinolone derivatives; Ultrasonic irradiation; One- pot synthesis; Green-sustainable perspectives

*Corresponding author: Mohamed Mokhtar (mmoustafa@kau.edu.sa; mmokhtar2000@yahoo.com); Tel.:+966500558045; Fax: +966-26952292

\section{Introduction}

Quinolone and their derivatives have several biological activities, such as anti-malaria [1], anticancer [2,3], anti-inflammatory [4], anti-bacterial [5], anti-asthmatic [6], anti-platelet activity [1,3, 4, 7], antihypertensive [8]. Therefore, several methods have been developed for the synthesis of quinolone derivatives utilizing various catalysts [9-12]. Recently, green and eco-friendly synthesis have attracted much attention. Numerous approaches have been established which improved green conditions to safer synthesis. ultrasonication is one of the auspicious green technology in the synthesis of organic compounds $[13,14]$. 
The use of nanoparticles (NPs) in catalysis is considered to be one of the most significant principles of green chemistry that is owing to a number of different reasons; the reaction time is short, diminishes generation of hazardous materials, economically visible as high yields produced with low cost [15]. nanoparticles have been widely used as the catalyst support in organic transformation [16]. Our previous achievements in the synthesis of different organic synthons of important biological activities utilizing different nanosized solid heterogeneous catalysts under green protocol [17-22] revealed that exploring efficient, sustainable and green catalyst is crucial to achieve green sustainable perspectives. To attain our goal, natural bio-polymer supported heterogeneous nano-catalysts that have been utilized in recent years $[23,24]$, was selected to be a catalyst support. One of the promising catalyst's support candidate is chitosan, which is produced by the $\mathrm{N}$-deacetylation of chitin. It is considered to be the second most abundant natural polymer after cellulose [25]. Chitosan a chemically stable, non-toxic is an excellent candidate to be used as a support for copper and other transition metals due to its insolubility in organic solvents and the presence of functionalize amino groups in the structure [26-28].

Nanoparticles have a special characteristic to aggregate and will clump together to form larger particles, thus nanoparticles lose their large surface area and other benefits [29]. Chitosan as a polymer-based stabilizes the nanoparticles to prevent their aggregation via coordination with metal nanoparticles through chelation mechanism, makes it a perfect support for metal nanoparticles [30].

Gold, silver and transition metals nanoparticles such as palladium are available for the development of hybrid catalyst complexes and they can also be used in chemical transformation whereas a new glyoxal cross-linked chitosan Schiff base was prepared as a support material for palladium catalyzed Suzuki cross coupling reactions [31,32]. In addition, chitosan was used as support of copper nanoparticles as catalyst for the C-S coupling of thiophenol with aryl halides [33]. The synthesis of $\mathrm{Cu}$ nanoparticles using chitosan as both reducing and capping agent was reported elsewhere [34,35]. This single step method is considered to be cost-effective, convenient and echo-friendly relative to other method of preparation [34].

In the present work, chitosan decorated copper nanoparticles catalysts were synthesized through green methods $[34,35]$ and its application as an efficient catalyst in multicomponent reaction to the synthesis of novel quinolone derivatives under ultrasonic irradiation were extensively studied. The Cu-CS-NPs catalyst is a promising efficient sustainable green catalyst for the synthesis of quinolone derivatives in satisfactory yield in short reaction time under ultrasonication conditions.

\section{Results}

\subsection{Catalytic Test}


The synthesis of quinoline derivatives via four-component one-pot reaction of p-chlorobenzaldehyde, dimedone, ethyl cyanoacetate and ammonium acetate is represented in scheme 1 . Neat reaction (absence of catalyst) showed 25\% yield after $6 \mathrm{~h}$ in ethanol under refluxing condition. The neat reaction (absence of catalyst) carried out under ultrasonic irradiation resulted in poor isolated yield of the product (29\%) in $15 \mathrm{~min}$. reaction time.

In order to find out suitable Cu-CS NPs catalyst for the synthesis of quinoline derivatives via fourcomponent one-pot reaction of P-Chlorobenzaldehyde, dimedone, ethyl cyanoacetate and ammonium acetate as the model reaction under ultrasound irradiation and its behavior was studied on the presence two kind of Cu-CS NPs catalyst. Firstly, has been studied the catalytic activity of model reaction via four weights from $\mathrm{Cu}-\mathrm{CS}$ NPs. then has been tested the catalytic activity of the model reaction also with using four weights from Cu-CS NPs /TPP on the same conditions. the weights of catalysts were as follows $(0.05,0.1,0.15,0.2)$. The results clearly showed that the best result was obtained at (Cu-Cs NPs $0.1 \mathrm{~g})$ provides high yield product Fig.6.

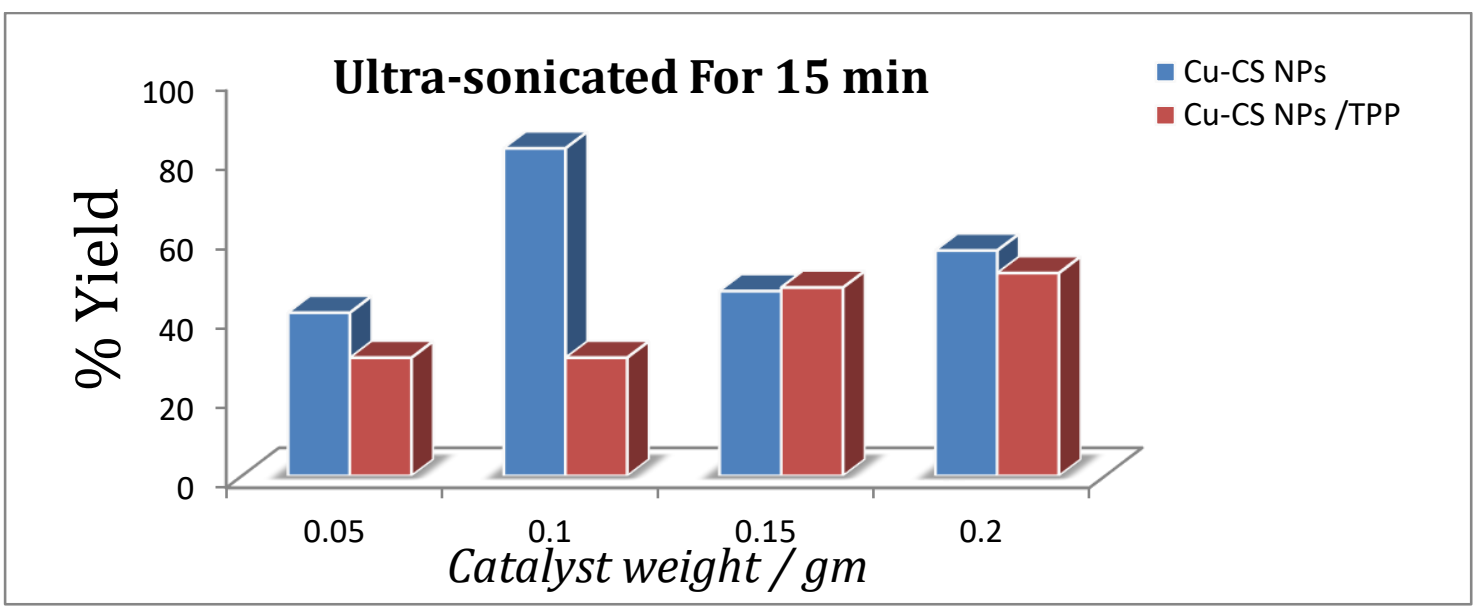

Figure 1. Catalytic activity of all the investigated catalysts with different masses.

The ultrasonic irradiation method has been chosen based on the advantages of this method, which is to shorten the time and also the slightly high product yield. Therefore, a variety of quinolines derivatives were also synthesized via this method by using the best catalyst (Cu-CS NPs $0.1 \mathrm{~g}$ ). After finding the best reaction condition, the reactions were carried out with various aldehydes under similar conditions. Catalyst provides an efficient synthesis of a new quinoline derivatives with several advantage involve high productivity, in short reaction time (Table1).<smiles>[R]C(=O)[O-]</smiles>

Scheme 1: Synthesis protocol of quinolone derivatives. 
Table 1: Effect of various aromatic aldehydes on the synthesis of polyhydroquinolines $2 \mathrm{a-j}$

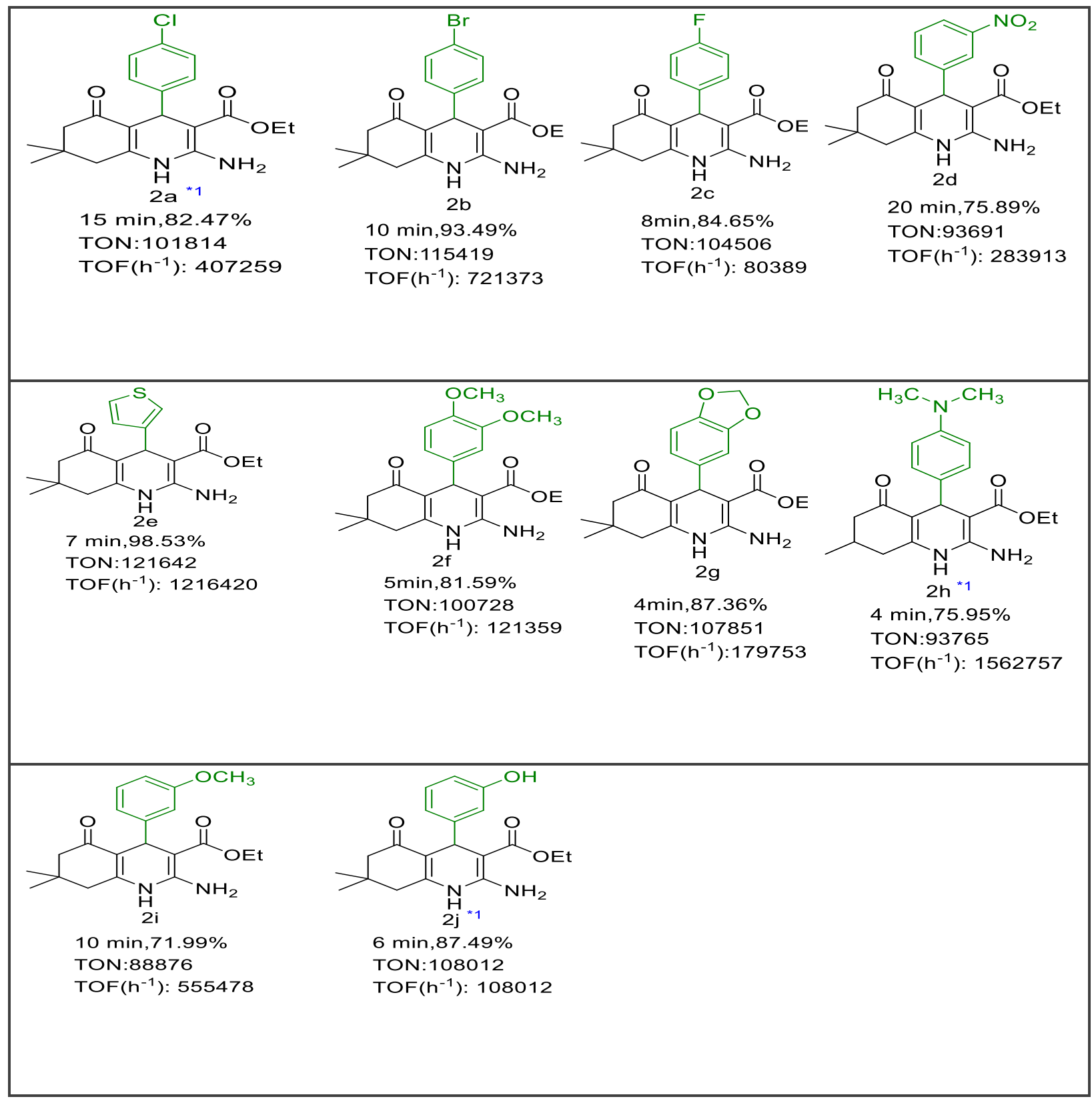

${ }^{{ }_{1} 1}$ S. Kumar, P. Sharma, K.K. Kapoor, M.S. Hundal, An efficient, catalyst-and solvent-free, four-component, and one-pot synthesis of polyhydroquinolines on grinding, Tetrahedron 64 (2008) 536-542.

As shown in table 1 the products were isolated in excellent yields. In addition, the reaction provides a new product with high TOF. Therefore, the catalyst plays crucial role in the success of the reaction in terms of rate and yields of polyhydroquinoline derivatives. Basically, catalytic activity of nanoparticles is related by size the particle so the surface of atom types changes with varying particle size. Moreover, the activity increases with decrease in particles size. On the other hand, the surface area of active species is increased. as well as spread the CuNPs on chitosan surface prevents the aggregation $\mathrm{Cu}$ NPs. $\mathrm{Cu}$ NPs appeared clearly in the TEM image (see characterization section).

Many catalysts have been used for one pot-catalytic synthesis of organic precursors utilizing nanocrystalline and nanoparticle catalysts such as $\mathrm{ClO}_{4} / \mathrm{Zr}-\mathrm{MCM}-41$ nanoparticles [36], $\mathrm{Fe}_{3} \mathrm{O}_{4} @ \mathrm{~B}-\mathrm{MCM}$ 41[37], and $\mathrm{ZnO}$ nanoparticles [38]. All of these catalysts showed pronounced catalytic activity due to 
their nanosized and large surface area features. In contrary to that the produced \% yield of products especially after re-use of catalysts for four time was not sufficient relative to our proposed catalyst in the present work. In order to study the sustainability of the present efficient catalyst towards four components one-pot catalytic synthesis of novel quinoline derivatives under ultrasound irradiation, the re-use test was carried out and the results are given in the following section.

\subsubsection{Reusability Procedure}

The reusability experiments were performed to investigate the stability of $\mathrm{Cu}$-CsNPs under the optimized reaction conditions using 4-chlorobenzaldehyde and dimedone as model substrates. Typically, after 15 in ultrasound irradiation, $\mathrm{Cu}$ - chitosan NPs was filtered and washed 4-6 times with hot ethanol to remove all the unreacted reactants and dried at room temperature for $24 \mathrm{~h}$. The dried $\mathrm{Cu}$-chitosan was used as catalyst in the subsequent runs and the results are summarized in Fig.2.

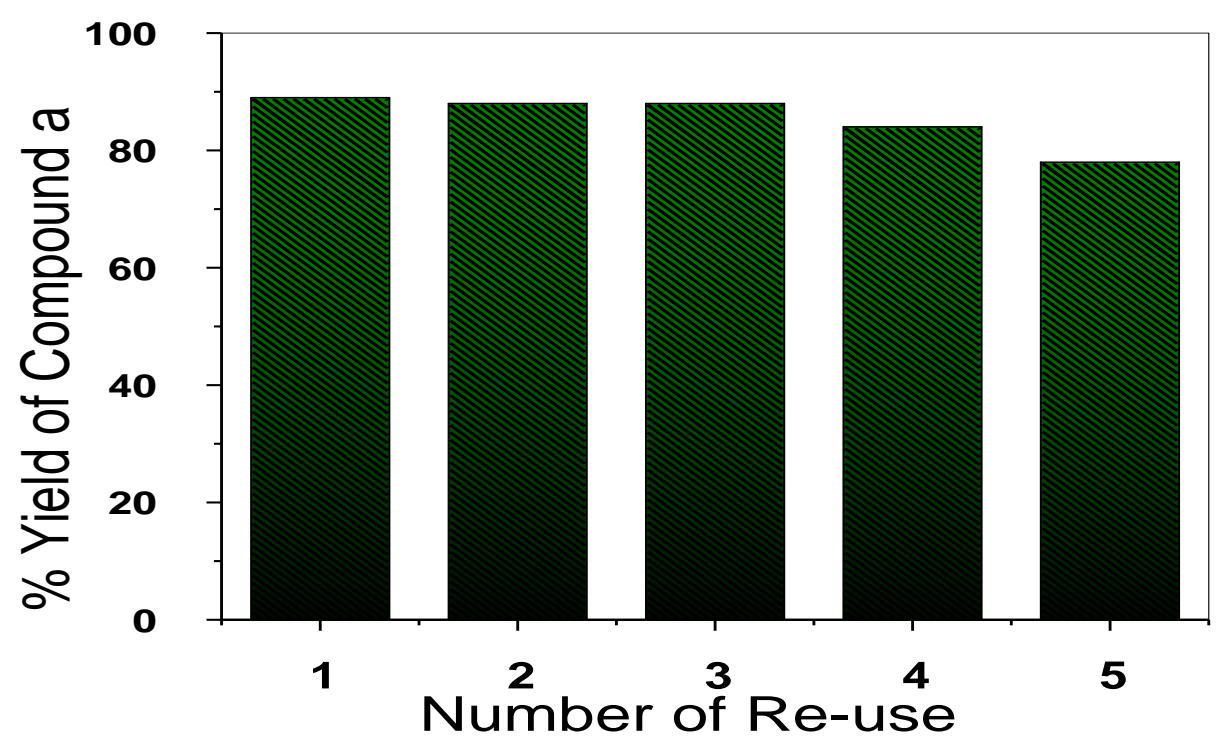

Figure 2. Robust feature of $\mathrm{Cu}-\mathrm{CS}$ A NPs catalyst after five times r-use

At the end of recycle tests, Cu-CS NPs catalyst had been five runs. The reusability test showed that promising catalyst could be re-used and regenerated for many times without substantial change in catalytic performance.

\subsection{Catalyst Characterization}

\subsubsection{FTIR of catalyst}

FT-IR spectra of chitosan and copper decorated chitosan nanoparticles (Fig.3) displayed a broad band for $\mathrm{OH}$ and $\mathrm{NH}$ stretching of amine groups located at $3250 \mathrm{~cm}^{-1}$. The existence of band at $1553 \mathrm{~cm}^{-1}$ is due to the presence of the $\mathrm{NH}_{2}$ groups. Stretching vibrations due to $\mathrm{C}-\mathrm{OH}$ and $\mathrm{C}-\mathrm{N}$ appeared consequently in the absorption bands in the range 1016 and $1402 \mathrm{~cm}^{-1}$. The absorption band placed at $2936 \mathrm{~cm}^{-1}$ is credited to the $\mathrm{C}-\mathrm{H}$ stretching mode of methylene groups [39]. The decoration of chitosan 
by copper nanoparticles resulted in the formation of new intense peaks in the fingerprint region at low -frequency (600-500 $\left.\mathrm{cm}^{-1}\right)$ due to formation of $\mathrm{Cu}-\mathrm{N}$ and $\mathrm{Cu}-\mathrm{O}$ coordinate bonds. Furthermore, the peak at $612 \mathrm{~cm}^{-1}$ assigns for CuNPs-chitosan interaction, indication that NPs were capped by the biopolymer [34].

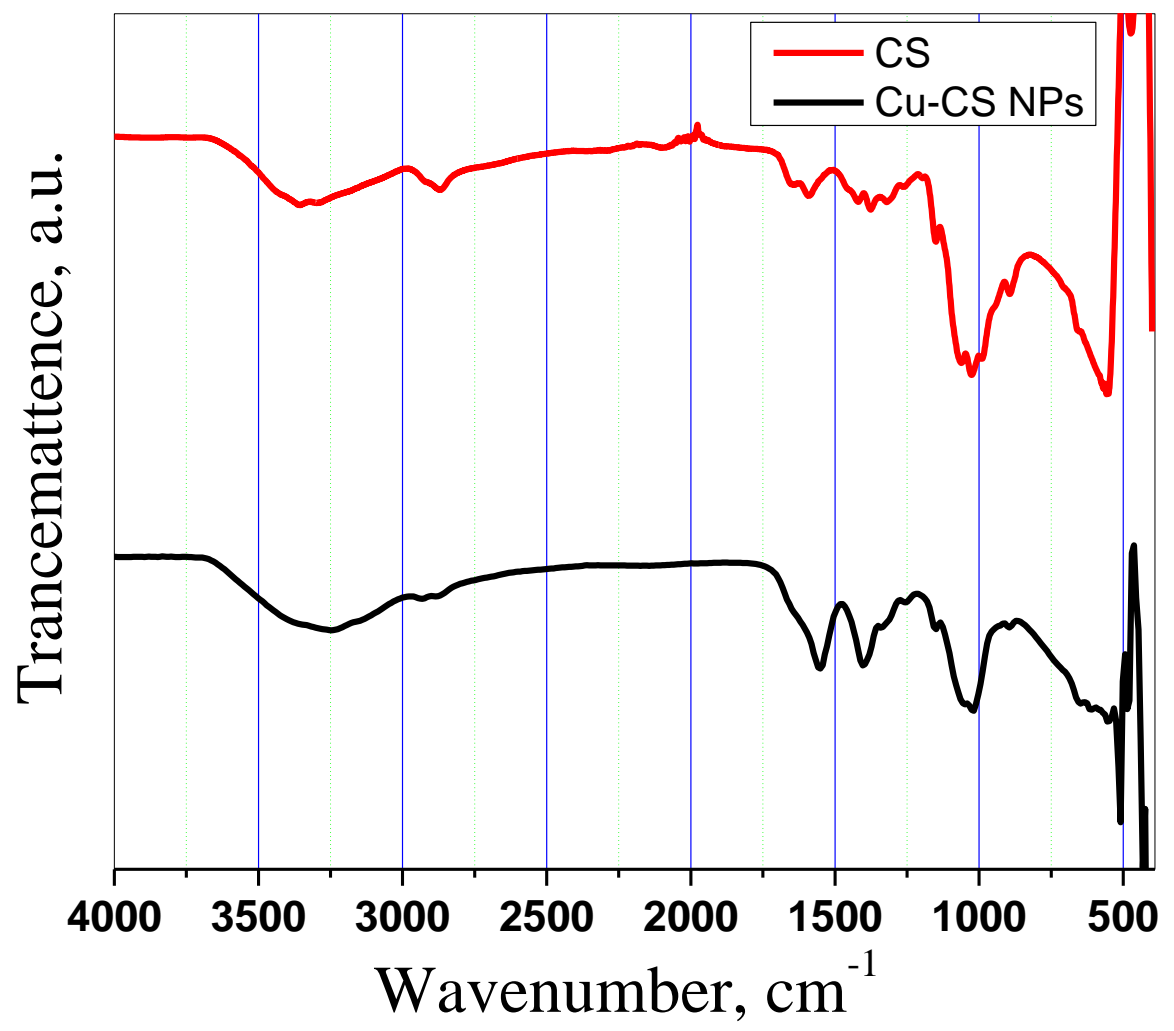

Figure 3. FTIR spectra of pure chitosan and chitosan decorated copper nanoparticles.

\subsubsection{XRD of investigated catalyst}

X-ray diffraction patterns of pure chitosan (CS) and copper nanoparticles decorated chitosan (Cu-CS NPs) are displayed in Figure 4. Some characteristic peaks for chitosan at $2 \theta=11.5^{\circ}$ and $22.5^{\circ}$ were observed $[40,41]$. A slight right shift with wider peak at $2 \theta=23^{\circ}$ recommends the decrease in crystallinity after anchoring copper nanoparticles in the chitosan structure. The main structure of chitosan was not disturbed with the absence of any characteristic peaks for copper nanoparticles. This observation suggests the dispersion of copper nanoparticles over the surface of chitosan and the structure of chitosan was not changed during the preparation method. 


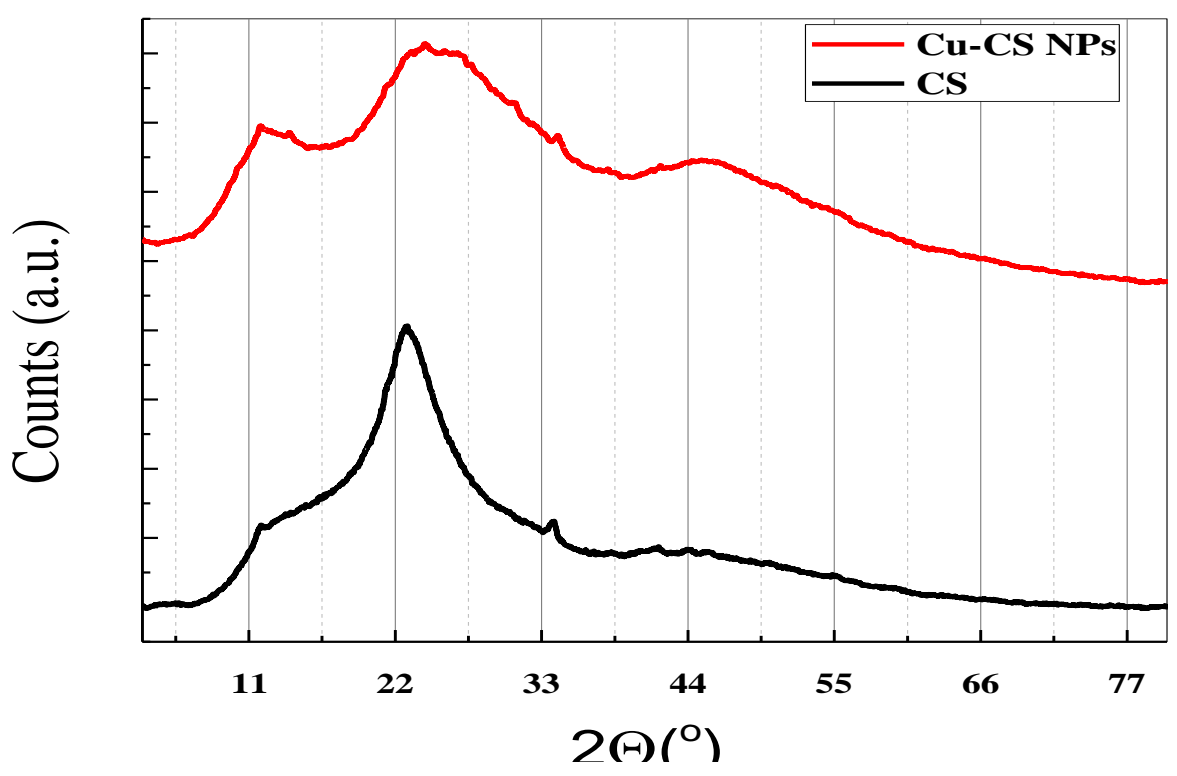

Figure 4. XRD patterns of chitosan (CS) and Cu-CS NPs

\subsubsection{SEM-EXD of Cu-Cs NPs catalyst}

The morphology of Cu-decorated chitosan sample (CuNPs) described by SEM image (Fig. 5) displayed asymmetrical deposits of chitosan. The nonattendance of copper nanoparticles could be ascribed to the good scattering of copper nanoparticles over chitosan. EDX spectra (Fig. 6) showed copper in addition to carbon, nitrogen and oxygen elements. The atomic \% of copper should be complemented by XPS analysis in order to give accurate turnover number of copper relative to the total atomic percentage derived from ICP-AES analysis.

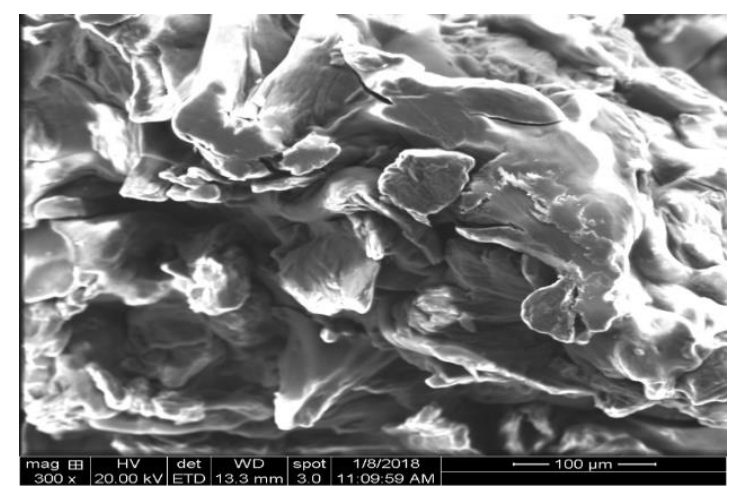

Figure 5. SEM images for Cu-CS NPs 


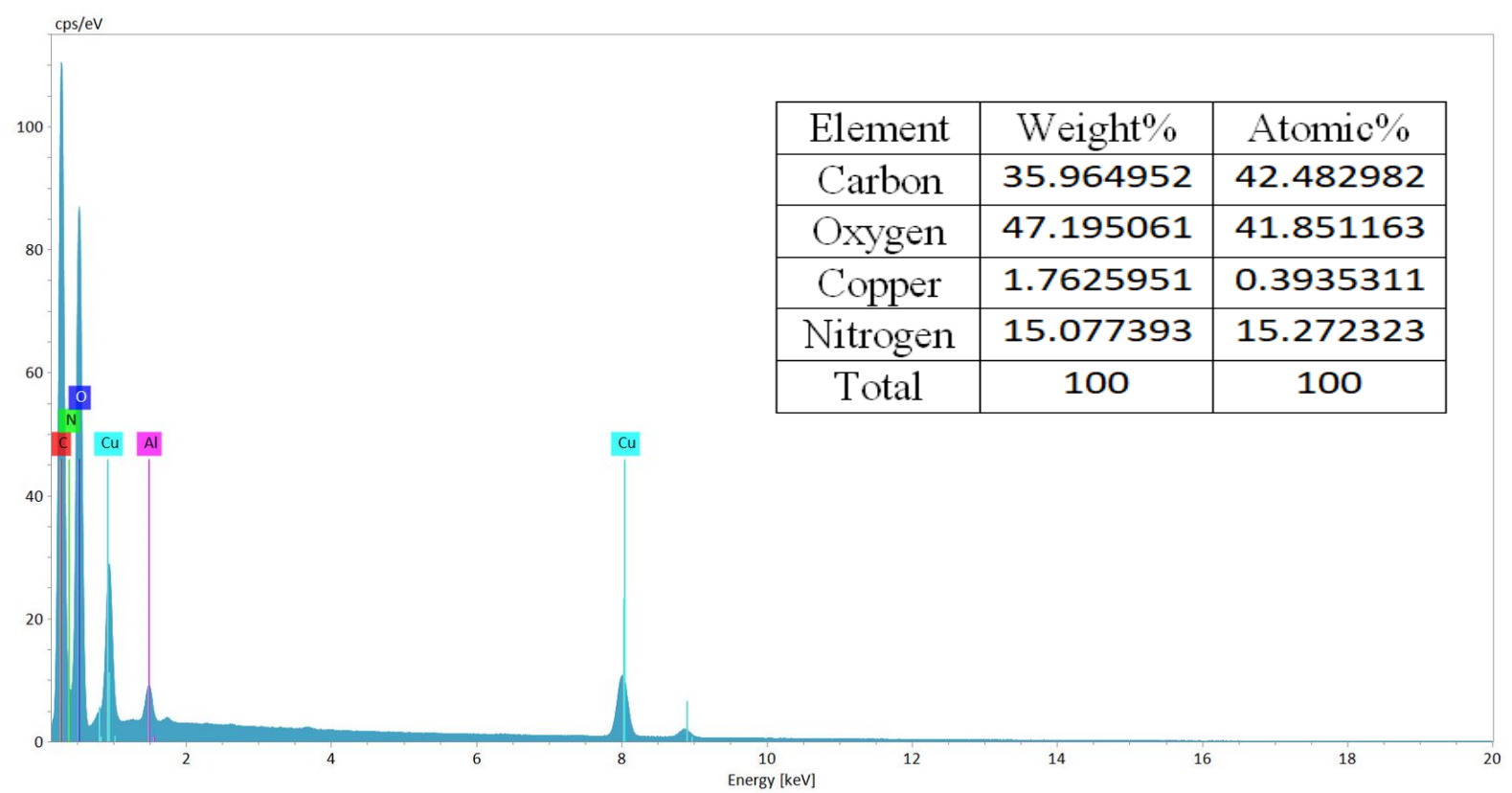

Figure 6. EDX spectra of copper decorated chitosan nanoparticles

\subsubsection{HRTEM and XPS of Cu-Cs NPs catalyst}

XPS peaks at 932.7 and $952.5 \mathrm{eV}$ corresponding to $\mathrm{Cu} 2 \mathrm{p} 3 / 2$ and $\mathrm{Cu} 2 \mathrm{p} 1 / 2$, respectively, which confirmed the presence of copper, which is not appeared in XRD patterns. Copper nanoparticles are fashioned in three diverse oxidation states, which could improve the catalytic efficacy of the synthesized catalyst. The number of moles of copper nanoparticles was detected from both EDX and XPS analyses for the determination of turnover number of active species. TEM images (Fig. 7 right-side) show copper nanoparticles are well dispersed over chitosan. The corresponding selected area diffraction (SAED pattern) shows uniform distribution of copper NPs in two faces (111) and (110).
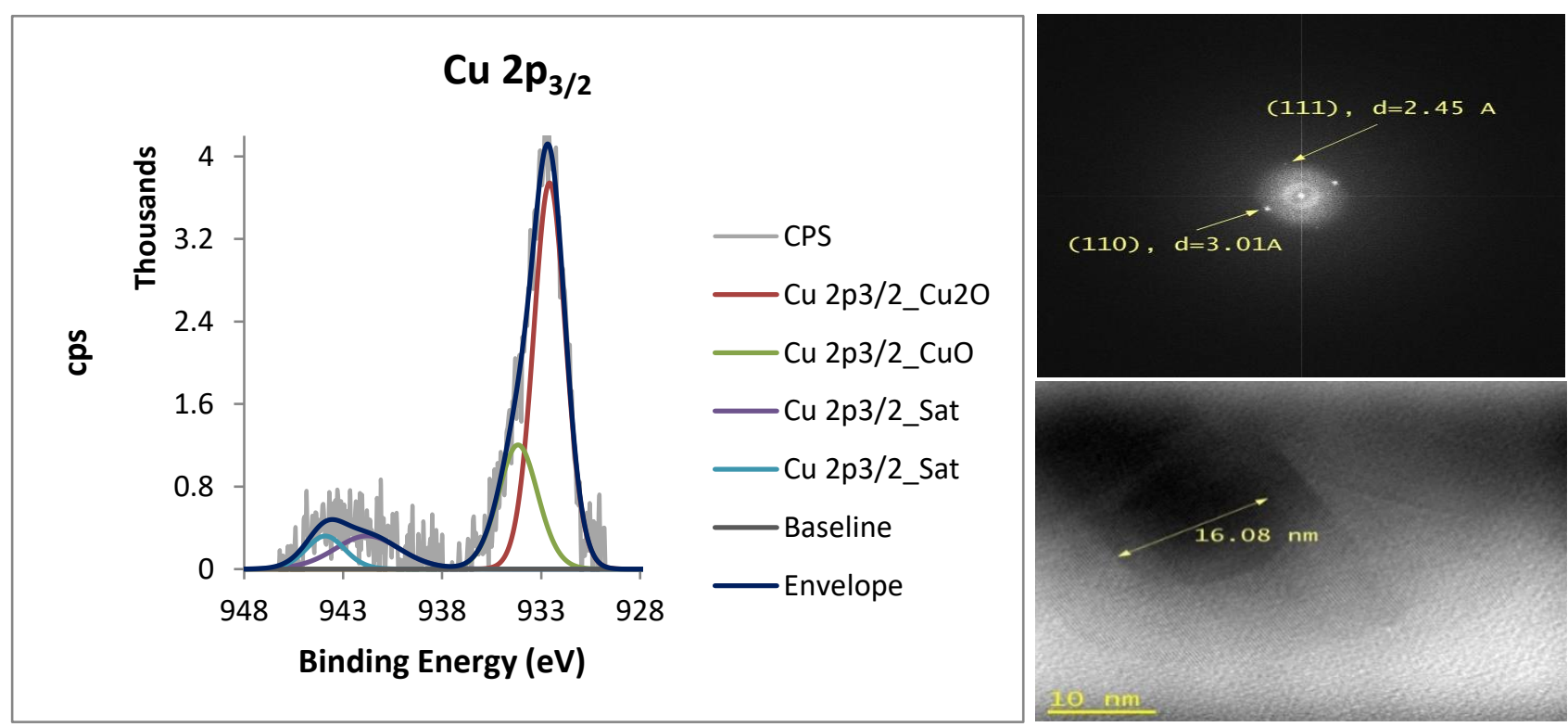

Figure 7. XPS (left-side) and HRTEM images (right-side) for Cu-CS NPs 


\subsection{Tentative Mechanism}

A tentative mechanism for multicomponent reaction of quinolines derivatives over Cu-Cs NPs has been proposed to occur via three different reaction steps (Scheme 2). Firstly, the well dispersed copper nanoparticles facilitate the electrophilicity of carbonyl group of the aldehyde via reduction of $\mathrm{Cu}^{2+}$ ions into $\mathrm{Cu}^{\mathrm{o}}$, which resulted in ease of attack on the active methylene carbon of ethylcyanoacetate and elimination of water. Secondly, the reaction proceeded via Michael addition assisted by Lewis acidic sites of the catalyst then followed by the last step in ionic mechanism.

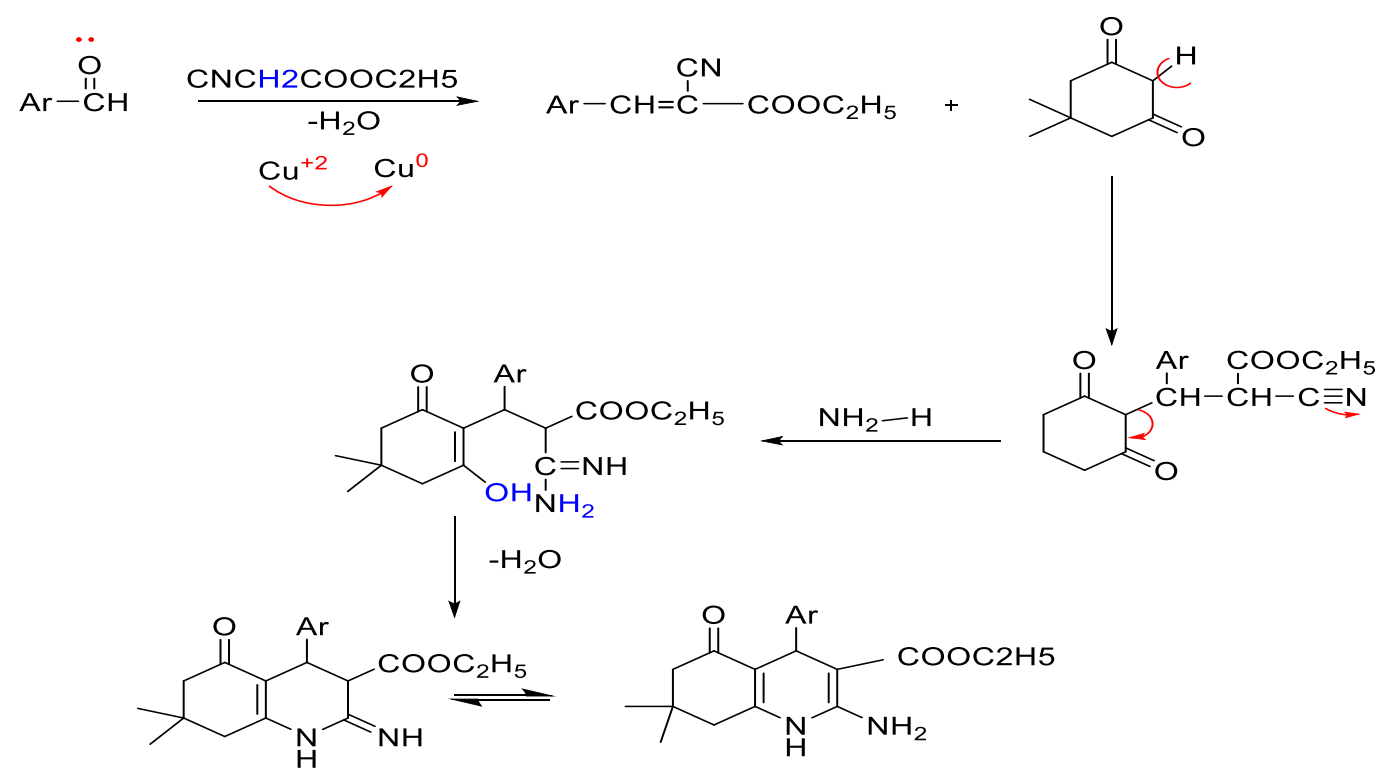

Scheme 2: Proposed mechanism for the synthesis of quinolone derivatives.

\section{Experimental details}

\subsection{Materials}

Chitosan (molecular weight 100,000-300,000) (Acros Organics- Belgium). Sodium tripolyphosphate (Acros Organics- Belgium), copper(II)acetate monohydrate (Central Drug House CDH, New Delhi, India) , dimedone, ethyl cyanoacetate, and ammonium acetate (Techno Pharm Chem, New Delhi, India). 4-chlorobenzaldehyde, 4-bromo benzaldehyde, 4-florobenzaldhyde, piperonal(1,3benzodioxole-5-carbaldehyde), salicylaldehyde (2-hydroxybenzaldehyde, thiophen -3-aldehyde,4dimethylaminobenzaldehyde, 2-methoxybenzaldhyde,3,4- dimethoxy benzaldehyde, m-nitro benzaldehyde (Merck KGaA, Darmstadt, Germany). Absolute ethanol and acetone (Fisher scientific, Leicestershire, U.K).

\subsection{Synthesis of Cu-Chitosan Catalyst}


The synthesis of $\mathrm{Cu}$ - chitosan nanoparticles was carried out according to two methods: Firstly: $\mathrm{Cu}$ chitosan NPs (Cu-Cs NPs) have been prepared via one-step synthesis green protocol. In a typical method, $0.75 \mathrm{~g}$ chitosan dissolving in $100 \mathrm{ml} 0.1 \%$ acetic acid (in distilled water) then $50 \mathrm{ml}$ of the solution and $25 \mathrm{ml}$ of $0.05 \mathrm{M}$ copper solution were delivered under stirring at $70^{\circ} \mathrm{C}$ for $9 \mathrm{~h}$ till the reaction was completed. the colloid was centrifuged for $10 \mathrm{~min}$. to separate particles from suspension then washed with acetone $(90 \%, v / v)$ and the centrifugation was repeated three times to remove unreacted reagents. The particles were dried under vacuum at the room temperature overnight and stored [34].

Secondly: Cu-chitosan NPs (Cu-Cs NPs /TPP) were prepared based on the ionotropic gelation between chitosan and sodium tripolyphosphate (TTP). Chitosan acted as a reducing/stabilizing agent. TPP was dissolved in water to a concentration of $0.25 \%$. Under magnetic stirring at room temperature, $33 \mathrm{ml}$ of TTP solution was added into $50 \mathrm{ml}$ of chitosan solution $0.75 \%$ (in dil. acetic acid $0.1 \%$ ) and the mixture was stirred for $15 \mathrm{~min}$.Chitosan nanoparticles loaded $\mathrm{Cu}^{2+}$ were obtained by adding metal ion solutions $16 \mathrm{ml} 0.05 \mathrm{M}$ into the chitosan nanosuspensions and heated to $70{ }^{\circ} \mathrm{C}$ using a water bath, after a blue color appeared, stirring continued for another $90 \mathrm{~min}$. before removing the heater. The resulting solution was cooled to room temperature for characterization [35].

\subsection{General procedure for the synthesis of polyhydroquinolines in the presence of Cu-chitosan}

A mixture of dimedone (3.6 mmol, $0.5 \mathrm{gm})$, aromatic aldehyde (3.6mmol, $0.5 \mathrm{gm})$, ethylcyanoacetoacetate $(3.6 \mathrm{mmol}, 0.38 \mathrm{ml})$, ammoniumacetate $(28.8 \mathrm{mmol}, 2.219 \mathrm{gm})$ and catalytic amounts of Cu-chitosan NPs $(0.1 \mathrm{gm})$ in $10 \mathrm{ml}$ absolute ethanol irradiate with ultrasonic waves at $80^{\circ} \mathrm{C}$. After completion of the reaction (monitored by TLC, petroleum ether: EtOAc, 1:2), the reaction mixture was filtered to separate the catalyst, then cooled at room temperature and the solid product obtained and was filtered off, dried and recrystallized from ethanol.

\subsection{Measurements and characterization}

The reactions were monitored by TLC and all yields refer to isolated products .Melting points were obtained by the Barnstead international 1002 melting point apparatus. IR spectra of the catalyst and products were recorded for the compounds in PerkinElmer spectrum 100 FT-IR spectrophotometer. ${ }^{1} \mathrm{H}$ NMR and ${ }^{13} \mathrm{C}$ spectra of products were recorded on Burker WM 400 and $850 \mathrm{MHz}$ spectrometer using TMS (0.00 ppm). Chemical shifts $(\delta)$ are given in ppm relative to the signal for TMS as standard, and coupling constants in Hz. PXRD patterns catalyst sample were analyzed using a Powder XRD diffractometer (Model Equinox1000 - INEL (France) with $\mathrm{Co} \mathrm{K}_{\alpha}(\lambda=1.7890 \AA$ ) radiation at $30 \mathrm{kV}$ and 
30mA. XPS measurements were carried out in an ultra-high vacuum multi- technique surface analysis system (SPECS GmbH, Germany) operating at a base pressure range of 1010 bar. Catalyst morphology was investigated by means of field emission scanning electron microscopy (FEG-SEM, Quanta FEG450, FEI, the Netherlands) using an ETD Everhart Thornley detector (High Vacuum mode), a solid-state backscattering electron detector (VCD)and EDS detector (XFLASH6-30, Brucker) for elemental analysis. HRTEM samples were prepared by sonication of the suspended powder in ethanol. A single drop of the sonicated suspension was deposit on TEM carbon grid 200 mesh and leaved for total evaporation at room temperature. Then the grid was mounted on a TEM single tilt holder, the residual solvent was removed by plasma cleaning process. The reactions that carried out by U.S irradiation was done using Daihan (Wiseclean, D-40 MHz) ultrasonic bath. Microanalysis was performed by Perkin Elmer elemental analyzer at the Faculty of Science, King Abdul Aziz University.

\subsection{Physical and spectroscopic data of product compounds}

Ethyl-2-amino-4-(4-chlorophenyl)-7,7-dimethyl-5-oxo-1,4,5,6,7,8hexahydroquinoline-3-carboxylate $(\mathrm{IV}$ )

Off - white crystals (1.09gm, $82.47 \%$ yield); m.p $173{ }^{\circ} \mathrm{C}$. FTIR; 3478,3328, $3200\left(-\mathrm{NH}, \mathrm{NH}_{2}\right) ; 1686,1655$ $(2 \mathrm{C}=\mathrm{O})$; and $1621 \mathrm{~cm}^{-1}(\mathrm{C}=\mathrm{C}) .{ }^{1} \mathrm{H}$ NMR $\left(400 \mathrm{MHz}, \mathrm{CDCl}_{3}\right): \delta_{\mathrm{H}}=0.96,1.09\left(6 \mathrm{H}, 2 \mathrm{~s}, 2 \mathrm{CH}_{3}\right) ; 1.14(3 \mathrm{H}, \mathrm{t},-$ $\left.\mathrm{CH}_{2} \mathrm{CH}_{3}, J=7.2 \mathrm{~Hz}\right) ; 1.84(1 \mathrm{H}$, br.s, $-\mathrm{NH}) ; 2.17\left(2 \mathrm{H}, \mathrm{dd}, \mathrm{C}_{8}-\mathrm{H}, \mathrm{J}=14 \mathrm{~Hz}\right) ; 2.41$ ( 2H, s , C6-H ); 4.03 (2H, q, $\left.\mathrm{CH}_{2} \mathrm{CH}_{3}, J=7.2 \mathrm{~Hz}\right) ; 4.66\left(1 \mathrm{H}, \mathrm{s}, \mathrm{C}_{4}-\mathrm{H}\right) ; 6.21\left(2 \mathrm{H}\right.$, br.s, $\left.\mathrm{NH}_{2}\right)$ and 7.15, $7.21(4 \mathrm{H}, 2 \mathrm{~d}, \mathrm{Ar}-\mathrm{H}) .{ }^{13} \mathrm{C} \mathrm{NMR}$ $\left(\mathrm{CDCl}_{3}\right): \delta_{\mathrm{c}}=14.22\left(\mathrm{CH}_{2} \underline{\mathrm{CH}_{3}}\right) ; 27.36,29.09\left(2 \mathrm{CH}_{3}\right) ; 32.24\left(\mathrm{C}_{7}\right) ; 33.48\left(\mathrm{C}_{4}\right) ; 40.66\left(\mathrm{C}_{8}\right) ; 50.69\left(\mathrm{C}_{6}\right) ; 59.77$ $\left(\underline{\mathrm{CH}_{2}} \mathrm{CH}_{3}\right) ; 80.30\left(\mathrm{C}_{3}\right) ; 116.40$ ( $\left.\mathrm{C}_{4 a}\right) ; 127.91$ ( $\left.\mathrm{C}_{3} \backslash, \mathrm{C}_{5} \backslash\right) ; 129.63\left(\mathrm{C}_{2} \backslash, \mathrm{C}_{6} \backslash\right) ; 131.63\left(\mathrm{C}_{4} \backslash\right) ; 144.46\left(\mathrm{C}_{1} \backslash\right) ; 158.37$ (C8a); $161.54,196.43$ (2 C=O); and $168.95\left(\mathrm{C}_{2}\right)$. Anal. Calcd. for $\mathrm{C}_{20} \mathrm{H}_{23} \mathrm{ClN}_{2} \mathrm{O}_{3}$ (374.86): C, 64.02; $\mathrm{H}, 6.61$; N, 7.47; O, 12.80. Found : C, 64.42; H, 6.43; N, 7.01; O, 12.58 .

Ethyl-2-amino-4-(4-bromophenyl)-7,7-dimethyl-5-oxo-1,4,5,6,7,8hexahydroquinoline-3-carboxylate $(\mathrm{IVb})$

Semisolid Off - white (1.41 gm, 93.49 \% yield). FTIR; 3473,3331,3204 (-NH, NH2); 1686,1654 (2C=O ); and $1620 \mathrm{~cm}^{-1}(\mathrm{C}=\mathrm{C}) .{ }^{1} \mathrm{H}$ NMR $\left(850 \mathrm{MHz}_{2} \mathrm{CDCl}_{3}\right): \delta_{\mathrm{H}}=0.96,1.09\left(6 \mathrm{H}, 2 \mathrm{~s}, 2 \mathrm{CH}_{3}\right) ; 1.15\left(3 \mathrm{H}, \mathrm{t},-\mathrm{CH}_{2} \underline{\mathrm{CH}_{3}}\right.$, $J=7.56 \mathrm{~Hz}) ; 2.08(1 \mathrm{H}, \mathrm{br} . \mathrm{s},-\mathrm{NH}) ; 2.18(2 \mathrm{H}, \mathrm{dd}, \mathrm{C} 8-\mathrm{H}, J=14 \mathrm{~Hz}) ; 2.42(2 \mathrm{H}, \mathrm{d} . \mathrm{d}, \mathrm{C} 6-\mathrm{H}, J=17 \mathrm{HZ}) ; 4.03(2 \mathrm{H}$, q, $\left.-\mathrm{CH}_{2} \mathrm{CH}_{3}, J=7.56 \mathrm{~Hz}\right) ; 4.65(1 \mathrm{H}, \mathrm{s}, \mathrm{C} 4-\mathrm{H}) ; 6.20\left(2 \mathrm{H}, \mathrm{br} . \mathrm{s}, \mathrm{NH}_{2}\right)$ and $7.13,7.33(4 \mathrm{H}, 2 \mathrm{~d}, \mathrm{Ar}-\mathrm{H}) .{ }^{13} \mathrm{C} \mathrm{NMR}$ $\left(\mathrm{CDCl}_{3}\right): \delta_{c}=14.21\left(\mathrm{CH}_{2} \underline{\mathrm{CH}_{3}}\right) ; 27.36,29.10\left(2 \mathrm{CH}_{3}\right) ; 32.25\left(\mathrm{C}_{7}\right) ; 33.44\left(\mathrm{C}_{4}\right) ; 40.63\left(\mathrm{C}_{8}\right) ; 50.65\left(\mathrm{C}_{6}\right) ; 59.79$ $\left(\underline{\mathrm{CH}_{2}} \mathrm{CH}_{3}\right) ; 80.22\left(\mathrm{C}_{3}\right) ; 116.30$ ( $\left.\mathrm{C}_{4 a}\right) ; 119.64\left(\mathrm{C}_{3} \backslash, \mathrm{C}_{5} \backslash\right) ; 130.07\left(\mathrm{C}_{2} \backslash, \mathrm{C}_{6} \backslash\right) ; 130.84\left(\mathrm{C}_{4} \backslash\right) ; 144.93\left(\mathrm{C}_{1} \backslash\right) ; 158.31$ (C8a); 161.55 ,196.46 (2 C=O); and $168.94\left(\mathrm{C}_{2}\right)$. Anal. Calcd. for $\mathrm{C}_{20} \mathrm{H}_{23} \mathrm{BrN}_{2} \mathrm{O}_{3}$ (419.31): C, 57.24; $\mathrm{H}, 4.48$; $\mathrm{N}, 6.68 ; \mathrm{O}, 11.45$. Found : $\mathrm{C}, 57.40 ; \mathrm{H}, 4.33 ; \mathrm{N}, 6.43 ; \mathrm{O}, 11.30$.

Ethyl-2-amino-4-(4-florophenyl)-7,7-dimethyl-5-oxo-1,4,5,6,7,8hexahydroquinoline-3-carboxylate ( IV $\left.{ }_{c}\right)$

Off - white crystals (1.08 gm, $84.65 \%$ yield); m.p $154{ }^{\circ} \mathrm{C}$. FTIR; 3398,3285, $3200\left(-\mathrm{NH}, \mathrm{NH}_{2}\right) ; 1689,1652$ $(2 \mathrm{C}=\mathrm{O})$; and $1601 \mathrm{~cm}^{-1}(\mathrm{C}=\mathrm{C}) .{ }^{1} \mathrm{H}$ NMR $\left(850 \mathrm{MHz}, \mathrm{CDCl}_{3}\right): \delta_{\mathrm{H}}=0.96,1.09\left(6 \mathrm{H}, 2 \mathrm{~s}, 2 \mathrm{CH}_{3}\right) ; 1.14(3 \mathrm{H}, \mathrm{t},-$ $\left.\mathrm{CH}_{2} \mathrm{CH}_{3}, J=7.65 \mathrm{~Hz}\right) ; 2.06$ (1H,br.s, $\left.-\mathrm{NH}\right) ; 2.17\left(2 \mathrm{H}, \mathrm{dd}, \mathrm{C}_{8}-\mathrm{H}, \mathrm{J}=16.15 \mathrm{~Hz}\right) ; 2.41$ ( 2H, d.d , C6-H , J= 17.83 $\mathrm{HZ}) ; 4.03\left(2 \mathrm{H}, \mathrm{q},-\mathrm{CH}_{2} \mathrm{CH}_{3}, J=6.8 \mathrm{~Hz}\right) ; 4.67\left(1 \mathrm{H}, \mathrm{s}, \mathrm{C}_{4}-\mathrm{H}\right) ; 6.26\left(2 \mathrm{H}, \mathrm{br} . \mathrm{s}, \mathrm{NH}_{2}\right)$ and 6.87, $7.23(4 \mathrm{H}, 2 \mathrm{~d}, \mathrm{Ar}-$ $\mathrm{H}) .{ }^{13} \mathrm{C} \mathrm{NMR}\left(\mathrm{CDCl}_{3}\right): \delta_{c}=14.21\left(\mathrm{CH}_{2} \underline{\mathrm{CH}_{3}}\right) ; 27.33,29.08\left(2 \mathrm{CH}_{3}\right) ; 32.23\left(\mathrm{C}_{7}\right) ; 33.24\left(\mathrm{C}_{4}\right) ; 40.62\left(\mathrm{C}_{8}\right) ; 50.68$ 
(C6); $59.71\left(\underline{\mathrm{CH}_{2}} \mathrm{CH}_{3}\right) ; 80.53\left(\mathrm{C}_{3}\right) ; 114.54\left(\mathrm{C}_{4 \mathrm{a}}\right) ; 116.61\left(\mathrm{C}_{3} \backslash, \mathrm{C}_{5} \backslash\right)$; $129.65\left(\mathrm{C}_{2} \backslash, \mathrm{C}_{6} \backslash\right)$; $141.65\left(\mathrm{C}_{4} \backslash\right)$; $158.32\left(\mathrm{C}_{1} \backslash\right)$; 160.65 (C8a); 161.42 ,196.53 (2 C=O); and 169.03 (C2) . Anal. Calcd. for $\mathrm{C}_{20} \mathrm{H}_{23} \mathrm{FN}_{2} \mathrm{O}_{3}$ (358.41): $\mathrm{C}, 66.96 ; \mathrm{H}$, 6.42; $\mathrm{N}, 7.81 ; \mathrm{O}, 13.39$. Found : $\mathrm{C}, 66.98 ; \mathrm{H}, 6.27 ; \mathrm{N}, 7.40 ; \mathrm{O}, 13.11$.

Ethyl-2-amino-4-(3-nitrophenyl)-7,7-dimethyl-5-oxo-1,4,5,6,7,8hexahydroquinoline-3-carboxylate (IVd)

Dark green crystals (1.04 gm, $75.89 \%$ yield); m.p $147^{\circ} \mathrm{C}$. FTIR; 3449,3332, $3200\left(-\mathrm{NH}, \mathrm{NH}_{2}\right) ; 1371\left(\mathrm{NO}_{2}\right)$; $1686,1657(2 \mathrm{C}=\mathrm{O})$; and $1621 \mathrm{~cm}^{-1}(\mathrm{C}=\mathrm{C}) .{ }^{1} \mathrm{H}$ NMR $\left(850 \mathrm{MHz}, \mathrm{CDCl}_{3}\right): \delta_{\mathrm{H}}=0.96,1.09\left(6 \mathrm{H}, 2 \mathrm{~s}, 2 \mathrm{CH}_{3}\right) ; 1.14$ $\left(3 \mathrm{H}, \mathrm{t},-\mathrm{CH}_{2} \mathrm{CH}_{3}, J=7.65 \mathrm{~Hz}\right) ; 2.10(1 \mathrm{H}, \mathrm{br} . \mathrm{s},-\mathrm{NH}) ; 2.17(2 \mathrm{H}, \mathrm{dd}, \mathrm{C} 8-\mathrm{H}, J=16.15 \mathrm{~Hz}) ; 2.47\left(2 \mathrm{H}, \mathrm{s}, \mathrm{C}_{6}-\mathrm{H}\right)$; $4.03\left(2 \mathrm{H}, \mathrm{q},-\mathrm{CH}_{2} \mathrm{CH}_{3}, J=6.8 \mathrm{~Hz}\right) ; 4.79\left(1 \mathrm{H}, \mathrm{s}, \mathrm{C}_{4}-\mathrm{H}\right) ; 6.31\left(2 \mathrm{H}\right.$, br.s, $\left.\mathrm{NH}_{2}\right)$ and $7.37\left(1 \mathrm{H}, \mathrm{d} . \mathrm{d}, \mathrm{C}_{5}-\mathrm{H}, J=7.65\right.$, $J=8.5 \mathrm{~Hz}$ of Ar) ; $7.64\left(1 \mathrm{H}, \mathrm{d}, \mathrm{C}_{4}-\mathrm{H}, J=7.65\right.$ of Ar $) ; 7.98\left(1 \mathrm{H}, \mathrm{d}, \mathrm{C}_{6}-\mathrm{H}, J=8.5 \mathrm{~Hz}\right.$ of Ar $) ; 8.09\left(1 \mathrm{H}, \mathrm{S}, \mathrm{C}_{2}-\mathrm{H}\right.$ of Ar ). $\left({ }^{13} \mathrm{C} \mathrm{NMR}\left(\mathrm{CDCl}_{3}\right)\right.$ : $\delta \mathrm{c}=14.19\left(\mathrm{CH}_{2} \mathrm{CH}_{3}\right) ; 27.37,29.06\left(2 \mathrm{CH}_{3}\right) ; 32.31\left(\mathrm{C}_{7}\right) ; 34.15\left(\mathrm{C}_{4}\right) ; 40.61\left(\mathrm{C}_{8}\right) ; 50.58$ $\left(\mathrm{C}_{6}\right) ; 59.91\left(\underline{\mathrm{CH}}_{2} \mathrm{CH}_{3}\right) ; 79.51\left(\mathrm{C}_{3}\right) ; 115.56\left(\mathrm{C}_{42}\right) ; 121.35\left(\mathrm{C}_{4} \backslash, \mathrm{C}_{6} \backslash\right)$; $123.16\left(\mathrm{C}_{5} !\right) ; 128.51\left(\mathrm{C}_{2}\right)$ ); $134.91\left(\mathrm{C}_{3} \backslash\right)$; $148.14\left(\mathrm{C}_{3}\right.$ ) $) ; 158.36\left(\mathrm{C}_{82}\right) ; 162.15,196.43(2 \mathrm{C}=\mathrm{O})$; and $168.66\left(\mathrm{C}_{2}\right)$. Anal. Calcd. for $\mathrm{C}_{20} \mathrm{H}_{23} \mathrm{~N}_{3} \mathrm{O}_{5}(385.41)$ : C, 62.27; H, 5.97; N, 10.89; O, 20.76 . Found : C, 62.48; H, 5.53; N, 10.45; O, 12.63.

Ethyl-2-amino-7,7-dimethyl-5-oxo-4-(thiophen-3-yl)-1,4,5,6,7,8-hexahydroquinoline-3-carboxylate $(\mathrm{IV}$ e)

Drak brown powder (1.22 gm, 98.53\% yield); m.p $123^{\circ} \mathrm{C}$. FTIR; 3427,3310, 3208 (-NH, NH$)$ ); 1688,1654 $(2 \mathrm{C}=\mathrm{O})$; and $1636 \mathrm{~cm}^{-1}(\mathrm{C}=\mathrm{C}) .{ }^{1} \mathrm{H}$ NMR $\left(850 \mathrm{MHz}, \mathrm{CDCl}_{3}\right): \delta_{\mathrm{H}}=1.01,1.12\left(6 \mathrm{H}, 2 \mathrm{~s}, 2 \mathrm{CH}_{3}\right) ; 1.20(3 \mathrm{H}, \mathrm{t},-$ $\left.\mathrm{CH}_{2} \mathrm{CH}_{3}, J=6.8 \mathrm{~Hz}\right) ; 2.13(1 \mathrm{H}, \mathrm{br} . \mathrm{s},-\mathrm{NH}) ; 2.26(2 \mathrm{H}, \mathrm{dd}, \mathrm{C} 8-\mathrm{H}, \mathrm{J}=16.15 \mathrm{~Hz}) ; 2.42\left(2 \mathrm{H}, \mathrm{s}, \mathrm{C}_{6}-\mathrm{H}\right) ; 4.10(2 \mathrm{H}$, q, $\left.-\mathrm{CH}_{2} \mathrm{CH}_{3}, J=7.65 \mathrm{~Hz}\right) ; 4.89\left(1 \mathrm{H}, \mathrm{s}, \mathrm{C}_{4}-\mathrm{H}\right) ; 6.19\left(2 \mathrm{H}, \mathrm{br} . \mathrm{s}, \mathrm{NH}_{2}\right)$ and $6.95(1 \mathrm{H}, \mathrm{d}, \mathrm{C} 5-\mathrm{H}$ of thiophene ), $7.10\left(1 \mathrm{H}, \mathrm{S}, \mathrm{C}_{2}-\mathrm{H}\right.$ of thiophene $), 7.12\left(1 \mathrm{H}, \mathrm{m}, \mathrm{C} 4-\mathrm{H}\right.$ of thiophene) $.{ }^{13} \mathrm{C} \mathrm{NMR}\left(\mathrm{CDCl}_{3}\right): \delta \mathrm{c}=14.18$ $\left(\mathrm{CH}_{2} \mathrm{CH}_{3}\right) ; 27.44,28.65\left(2 \mathrm{CH}_{3}\right) ; 32.24\left(\mathrm{C}_{7}\right) ; 40.67\left(\mathrm{C}_{8}\right) ; 41.21\left(\mathrm{C}_{4}\right) ; 50.74\left(\mathrm{C}_{6}\right) ; 59.73\left(\underline{\mathrm{CH}}_{2} \mathrm{CH}_{3}\right) ; 80.38\left(\mathrm{C}_{3}\right) ;$ 116.61 ( $\left.\mathrm{C}_{4 a}\right) ; 120.92\left(\mathrm{C}_{3} !\right) ; 124.55\left(\mathrm{C}_{2} !\right)$; $127.71\left(\mathrm{C}_{4} !\right)$; $147.76\left(\mathrm{C}_{1}{ }^{\prime}\right) ; 158.73\left(\mathrm{C}_{8 \mathrm{a}}\right) ; 162.03,197.34(2 \mathrm{C}=\mathrm{O})$; and $169.35\left(\mathrm{C}_{2}\right)$. Anal. Calcd. for $\mathrm{C}_{18} \mathrm{H}_{22} \mathrm{~N}_{2} \mathrm{O}_{3} \mathrm{~S}(346.44)$ : $\mathrm{C}, 62.35 ; \mathrm{H}, 6.35 ; \mathrm{N}, 8.08 ; \mathrm{O}, 13.85 ; \mathrm{S}, 9.24$. Found : C, 62.22; H, 6.16; N, 8.01; O, 13.46; S, 9.17.

Ethyl-2-amino-4-(3,4-dimethoxyphenyl)-7,7-dimethyl-5-oxo-1,4,5,6,7,8-hexahydroquinoline-3carboxylate $\left(\mathrm{IV}_{\mathrm{f}}\right)$

Off - white powder (1.17 gm, $81.59 \%$ yield); m.p $128^{\circ} \mathrm{C}$. FTIR; 3429,3314, $3200\left(-\mathrm{NH}, \mathrm{NH}_{2}\right) ; 1687,1663$ $(2 \mathrm{C}=\mathrm{O})$; and $1588 \mathrm{~cm}^{-1}(\mathrm{C}=\mathrm{C}) .{ }^{1} \mathrm{H}$ NMR $\left(850 \mathrm{MHz}, \mathrm{CDCl}_{3}\right): \mathrm{\delta H}_{\mathrm{H}}=0.98,1.10(6 \mathrm{H}, 2 \mathrm{~s}, 2 \mathrm{CH}) ; 1.19(3 \mathrm{H}, \mathrm{t},-$ $\left.\mathrm{CH}_{2} \mathrm{CH}_{3}, J=6.8 \mathrm{~Hz}\right) ; 2.13$ (1H,br.s, $\left.-\mathrm{NH}\right) ; 2.19$ (2H, dd, $\left.\mathrm{C}_{8}-\mathrm{H}, J=16.9 \mathrm{~Hz}\right) ; 2.41$ ( 2H, d.d , C6- $\mathrm{C}_{1} J=17.85$ $\mathrm{Hz}) ; 3.96,3.97\left(6 \mathrm{H}, 2 \mathrm{~S}, 2-\mathrm{OCH}_{3}\right) ; 4.38\left(2 \mathrm{H}, \mathrm{q},-\mathrm{CH}_{2} \mathrm{CH}_{3}, J=7.65 \mathrm{~Hz}\right) ; 4.65\left(1 \mathrm{H}, \mathrm{s}, \mathrm{C}_{4}-\mathrm{H}\right) ; 6.69\left(2 \mathrm{H}\right.$, br.s, $\left.\mathrm{NH}_{2}\right)$ and 7.74,7.80,8.16 (3H, d.d, s, Ar-H). ${ }^{13} \mathrm{C} \mathrm{NMR}\left(\mathrm{CDCl}_{3}\right)$ : $\delta \mathrm{c}=14.22\left(\mathrm{CH}_{2} \mathrm{CH}_{3}\right) ; 27.30,29.27\left(2 \mathrm{CH}_{3}\right) ; 32.24$ $\left(\mathrm{C}_{7}\right) ; 33.29\left(\mathrm{C}_{4}\right) ; 40.67\left(\mathrm{C}_{8}\right) ; 50.81\left(\mathrm{C}_{6}\right) ; 55.65,56.07\left(2-\mathrm{O}_{\mathbf{C H}}\right)$ ) $59.69\left(\underline{\mathrm{CH}}_{2} \mathrm{CH}_{3}\right) ; 80.92\left(\mathrm{C}_{3}\right) ; 110.995$ ( $\left.\mathrm{C}_{4 a}\right)$

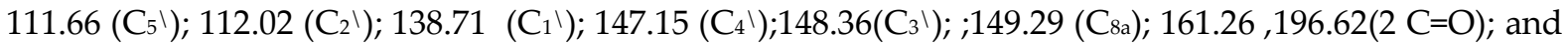
163.13(C2) . Anal. Calcd. for $\mathrm{C}_{22} \mathrm{H}_{28} \mathrm{~N}_{2} \mathrm{O}_{5}$ (400.47): C, 65.92; H, 6.99; N, 6.99; O, 19.98. Found : $\mathrm{C}, 65.99 ; \mathrm{H}$, $6.61 ; \mathrm{N}, 6,36 ; \mathrm{O}, 19.44$.

Ethyl- 2-amino-4-(benzo[d][1,3]dioxol-5-yl)-7,7-dimethyl-5-oxo-1,4,5,6,7,8-hexahydroquinoline-3carboxylate $\left(\mathrm{IV}_{\mathrm{g}}\right)$

Off - white crystals $\left(1.20\right.$ gm, $87.36 \%$ yield); m.p $133^{\circ} \mathrm{C}$. FTIR; 3437, $3204\left(-\mathrm{NH}, \mathrm{NH}_{2}\right) ; 1688,1653(2 \mathrm{C}=\mathrm{O}$ ); and $1606 \mathrm{~cm}^{-1}(\mathrm{C}=\mathrm{C}) .{ }^{1} \mathrm{H}$ NMR $\left(850 \mathrm{MHz}, \mathrm{CDCl}_{3}\right): \mathrm{\delta H}_{\mathrm{H}}=0.99,1.09\left(6 \mathrm{H}, 2 \mathrm{~s}, 2 \mathrm{CH}_{3}\right) ; 1.18\left(3 \mathrm{H}, \mathrm{t},-\mathrm{CH}_{2} \mathrm{CH}_{3}\right.$, $J=7.65 \mathrm{~Hz}) ; 2.08(1 \mathrm{H}, \mathrm{br} . \mathrm{s},-\mathrm{NH}) ; 2.19\left(2 \mathrm{H}, \mathrm{dd}, \mathrm{C}_{8}-\mathrm{H}, J=16.15 \mathrm{~Hz}\right) ; 2.41\left(2 \mathrm{H}, \mathrm{s}, \mathrm{C}_{6}-\mathrm{H}\right) ; 4.36(2 \mathrm{H}, \mathrm{q},-$ $\left.\mathrm{CH}_{2} \mathrm{CH}_{3}, J=6.8 \mathrm{~Hz}\right) ; 4.62\left(1 \mathrm{H}, \mathrm{s}, \mathrm{C}_{4}-\mathrm{H}\right) ; 5.87\left(2 \mathrm{H}, 2 \mathrm{~d},-\mathrm{O}-\mathrm{CH}_{2}-\mathrm{O}\right) ; 6.08\left(2 \mathrm{H}, \mathrm{br} . \mathrm{s}, \mathrm{NH}_{2}\right)$ and 6.65,6.74,6.90(3H, d,d.d,d, Ar-H). ${ }^{13} \mathrm{C}$ NMR $\left(\mathrm{CDCl}_{3}\right)$ : $\delta \mathrm{c}=14.28\left(\mathrm{CH}_{2} \mathrm{CH}_{3}\right) ; 27.52,29.04\left(2 \mathrm{CH}_{3}\right) ; 32.25\left(\mathrm{C}_{7}\right) ; 33.29\left(\mathrm{C}_{4}\right) ; 40.64$

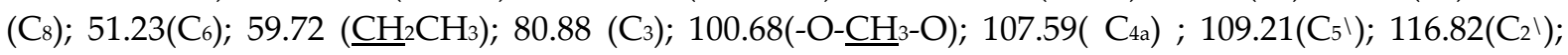


121.34 ( $\left.\mathrm{C}_{6}{ }^{\prime}\right) ; 139.98\left(\mathrm{C}_{1}{ }^{\prime}\right) ; 145.65\left(\mathrm{C}_{4}{ }^{\prime}\right)$; 147.07( $\left.\mathrm{C}_{3}{ }^{\prime}\right) ; 158.20$ ( $\left.\mathrm{C}_{8 \mathrm{a}}\right) ; 162.99,196.56$ (2 C=O); and $169.11\left(\mathrm{C}_{2}\right)$. Anal. Calcd. for $\mathrm{C}_{21} \mathrm{H}_{24} \mathrm{~N}_{2} \mathrm{O}_{5}$ (384.17): $\mathrm{C}, 65.59 ; \mathrm{H}, 6.25 ; \mathrm{N}, 7.29 ; \mathrm{O}, 20.82$. Found : $\mathrm{C}, 65.92 ; \mathrm{H}, 6.13 ; \mathrm{N}$, 7.01; O, 20.54 .

Ethyl-2-amino-4-(2-dimethylamino(phenyl)-7,7-dimethyl-5-oxo-1,4,5,6,7,8hexahydroquinoline-3carboxylate $(\mathrm{IVh})$

Light yellow crystals (1.04 gm, $75.95 \%$ yield); m.p $115^{\circ} \mathrm{C}$. FTIR; 3196,2932, $3200\left(-\mathrm{NH}, \mathrm{NH}_{2}\right) ; 1702,1610$ $(2 \mathrm{C}=\mathrm{O})$; and $1561 \mathrm{~cm}^{-1}(\mathrm{C}=\mathrm{C}) .{ }^{1} \mathrm{H}$ NMR $\left(850 \mathrm{MHz}, \mathrm{CDCl}_{3}\right): \mathrm{\delta H}_{\mathrm{H}}=1.08\left(6 \mathrm{H}, 2 \mathrm{~s}, 2 \mathrm{CH}_{3}\right) ; 1.38\left(3 \mathrm{H}, \mathrm{t},-\mathrm{CH}_{2} \mathrm{CH}_{3}\right.$, $J=6.8 \mathrm{~Hz}) ; 1.61(1 \mathrm{H}$, br.s, $-\mathrm{NH}) ; 2.14(2 \mathrm{H}, \mathrm{dd}, \mathrm{C} 8-\mathrm{H}, J=14 \mathrm{~Hz}) ; 2.26\left(2 \mathrm{H}, \mathrm{s}, \mathrm{C}_{6}-\mathrm{H}\right) ; 3.09(6 \mathrm{H}, \mathrm{s}, \mathrm{N}(\mathrm{C} \underline{\mathrm{H} 3}) 2)$ ;4.34 (2H, q, $\left.-\mathrm{CH}_{2} \mathrm{CH}_{3}, J=7.65 \mathrm{~Hz}\right) ; 4.98\left(1 \mathrm{H}, \mathrm{s}, \mathrm{C}_{4}-\mathrm{H}\right) ; 6.64\left(2 \mathrm{H}\right.$, br.s, $\left.\mathrm{NH}_{2}\right)$ and 6.70, $7.94(4 \mathrm{H}, 2 \mathrm{~d}, \mathrm{Ar}-\mathrm{H})$. ${ }^{13} \mathrm{C} \mathrm{NMR}\left(\mathrm{CDCl}_{3}\right): \delta \mathrm{c}=14.30\left(\mathrm{CH}_{2} \underline{\mathrm{CH}}_{3}\right) ; 27.38,28.34\left(2 \mathrm{CH}_{3}\right) ; 32.90\left(\mathrm{C}_{7}\right) ; 39.98\left(\mathrm{C}_{4}\right) ; 40.03\left(\mathrm{C}_{8}\right) ; 41.40,42.64$ $\left(\mathrm{N}-(\underline{\mathrm{CH}} 3)_{2}\right), 50.72\left(\mathrm{C}_{6}\right) ; 61.58\left(\underline{\mathrm{CH}}_{2} \mathrm{CH}_{3}\right) ; 94.06\left(\mathrm{C}_{3}\right) ; 110.09$ ( $\left.\mathrm{C}_{4 a}\right) ; 111.50,112.67\left(\mathrm{C}_{2} \backslash, \mathrm{C}_{6}\right.$ । $) ; 117.60,119.81$ $\left.\left.\left(\mathrm{C}_{3} \backslash, \mathrm{C}_{5}\right)^{\prime}\right) ; 134.07\left(\mathrm{C}_{1}\right)^{\prime}\right) ; 136.07\left(\mathrm{C}_{1}{ }^{\prime}\right) ; 154.58\left(\mathrm{C}_{8 \mathrm{a}}\right) ; 163.15,197.59$ (2 C=O); and 164.32 ( $\left.\mathrm{C}_{2}\right)$. Anal. Calcd. for $\mathrm{C}_{22} \mathrm{H}_{29} \mathrm{~N}_{3} \mathrm{O}_{3}$ (383.48): $\mathrm{C}, 68.84 ; \mathrm{H}, 7.56 ; \mathrm{N}, 10.95 ; \mathrm{O}, 12.52$. Found : $\mathrm{C}, 68.92 ; \mathrm{H}, 7.32 ; \mathrm{N}, 10.61 ; \mathrm{O}, 12.17$.

Ethyl-2-amino-4-(2-methoxyphenyl)-7,7-dimethyl-5-oxo-1,4,5,6,7,8hexahydroquinoline-3-carboxylate $\left(\mathrm{IV}_{\mathrm{i}}\right)$

Dark green crystals (0.96 gm, 71.99\% yield); m.p $185{ }^{\circ} \mathrm{C}$. FTIR; 3421,3309, 3200 (-NH, NH$)$; 1685,1649 $(2 \mathrm{C}=\mathrm{O})$; and $1613 \mathrm{~cm}^{-1}(\mathrm{C}=\mathrm{C}) .{ }^{1} \mathrm{H}$ NMR $\left(400 \mathrm{MHz}, \mathrm{CDCl}_{3}\right): \delta_{\mathrm{H}}=0.94,1.08\left(6 \mathrm{H}, 2 \mathrm{~s}, 2 \mathrm{CH}_{3}\right) ; 1.15(3 \mathrm{H}, \mathrm{t},-$ $\left.\mathrm{CH}_{2} \mathrm{CH}_{3}, J=7.65 \mathrm{~Hz}\right) ; 2.08$ (1H,br.s, $\left.-\mathrm{NH}\right) ; 2.13\left(2 \mathrm{H}, \mathrm{dd}, \mathrm{C}_{8}-\mathrm{H}, J=17 \mathrm{~Hz}\right) ; 2.41$ ( 2 $\mathrm{H}, \mathrm{dd}, \mathrm{C}_{6}-\mathrm{H}, J=16.15 \mathrm{~Hz}$ ); $3.75\left(3 \mathrm{H}, \mathrm{S},-\mathrm{OCH}_{3}\right), 4.00\left(2 \mathrm{H}, \mathrm{q},-\mathrm{CH}_{2} \mathrm{CH}_{3}, J=6.8 \mathrm{~Hz}\right) ; 4.77\left(1 \mathrm{H}, \mathrm{s}, \mathrm{C}_{4}-\mathrm{H}\right) ; 6.48\left(2 \mathrm{H}\right.$, br.s, $\left.\mathrm{NH}_{2}\right)$ and 6.76,6.83, 7.09,7.32 (4H, d,dd,d,dd Ar-H). ${ }^{13} \mathrm{C} \mathrm{NMR}\left(\mathrm{CDCl}_{3}\right): \delta \mathrm{c}=14.18\left(\mathrm{CH}_{2} \underline{\mathrm{CH}}_{3}\right) ; 26.87,29.35\left(2 \mathrm{CH}_{3}\right)$; $31.61\left(\mathrm{C}_{4}\right) ; 32.15\left(\mathrm{C}_{7}\right) ; 40.74\left(\mathrm{C}_{8}\right) ; 50.73\left(\mathrm{C}_{6}\right) ; 55.20\left(-\mathrm{OCH}_{3}\right) ; 59.46\left(\underline{\mathrm{CH}}_{2} \mathrm{CH}_{3}\right) ; 78.94\left(\mathrm{C}_{3}\right) ; 110.72\left(\mathrm{C}_{4 a}\right)$; 114.57( $\left.\mathrm{C}_{3} \backslash\right)$; $119.82\left(\mathrm{C}_{1} \backslash, \mathrm{C}_{5} \backslash\right)$; $127.37\left(\mathrm{C}_{4} \backslash\right) ; 132.03\left(\mathrm{C}_{6} \backslash\right) ; 157.79\left(\mathrm{C}_{8 \mathrm{a}}\right) ; 158.91\left(\mathrm{C}_{2}{ }^{\prime}\right) ; 162.10,196.70(2 \mathrm{C}=\mathrm{O})$; and $169.57\left(\mathrm{C}_{2}\right)$. Anal. Calcd. for $\mathrm{C}_{21} \mathrm{H}_{26} \mathrm{~N}_{2} \mathrm{O}_{4}$ (370.44): $\mathrm{C}, 68.03 ; \mathrm{H}, 7.02 ; \mathrm{N}, 7.56 ; \mathrm{O}, 17.28$. Found : $\mathrm{C}$, 68.44; H, 6.99; N, 7.05; O, 17.01 .

Ethyl2-amino-4-(2-hydroxyphenyl)-7,7-dimethyl-5-oxo-1,4,5,6,7,8hexahydroquinoline-3-carboxylate $\left(\mathrm{IV}_{\mathrm{j}}\right)$

Yellow powder (1.11 gm, $87.49 \%$ yield); m.p $114{ }^{\circ} \mathrm{C}$. FTIR; 3600-2900 (br.band for -NH, NH $2, \mathrm{OH}$ ); $1705,1655(2 \mathrm{C}=\mathrm{O})$; and $1630 \mathrm{~cm}^{-1}(\mathrm{C}=\mathrm{C}) .{ }^{1} \mathrm{H}$ NMR $\left(400 \mathrm{MHz}, \mathrm{CDCl}_{3}\right): \delta_{\mathrm{H}}=0.99\left(6 \mathrm{H}, 2 \mathrm{~s}, 2 \mathrm{CH}_{3}\right) ; 1.08(3 \mathrm{H}$, t, $\left.-\mathrm{CH}_{2} \mathrm{CH}_{3}, J=7.2 \mathrm{~Hz}\right) ; 1.18(1 \mathrm{H}, \mathrm{br} . \mathrm{s},-\mathrm{NH}) ; 2.53\left(2 \mathrm{H}, \mathrm{dd}, \mathrm{C}_{8}-\mathrm{H}, J=14 \mathrm{~Hz}\right) ; 2.66$ ( 2H, s , C6- $\left.\mathrm{H}\right) ; 3.90(2 \mathrm{H}$, q, $\left.-\mathrm{CH}_{2} \mathrm{CH}_{3}, J=7.2 \mathrm{~Hz}\right) ; 4.67\left(1 \mathrm{H}, \mathrm{s}, \mathrm{C}_{4}-\mathrm{H}\right) ; 6.45\left(2 \mathrm{H}\right.$, br.s, $\left.\mathrm{NH}_{2}\right)$ and 7.01,7.16,7.26,7.86 $(4 \mathrm{H}, \mathrm{dd}, \mathrm{dd}, 2 \mathrm{~d}, \mathrm{Ar}-$ H). ${ }^{13} \mathrm{C} \mathrm{NMR}\left(\mathrm{CDCl}_{3}\right)$ : $\delta \mathrm{c}=14.05\left(\mathrm{CH}_{2} \underline{\mathrm{CH}}_{3}\right) ; 27.21,27.78\left(2 \mathrm{CH}_{3}\right) ; 32.32\left(\mathrm{C}_{7}\right) ; 32.58\left(\mathrm{C}_{4}\right) ; 42.55\left(\mathrm{C}_{8}\right) ; 47.88$

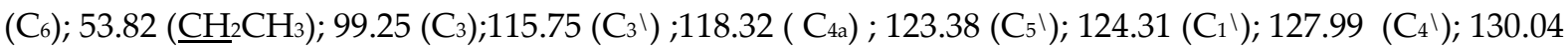
$\left(\mathrm{C}_{1} \backslash\right) ; 147.64\left(\mathrm{C}_{8 \mathrm{a}}\right) ; 151.61\left(\mathrm{C}_{2} \backslash\right) ; 161.48,197.35(2 \mathrm{C}=\mathrm{O})$; and $169.73\left(\mathrm{C}_{2}\right)$. Anal. Calcd. for $\mathrm{C}_{20} \mathrm{H}_{24} \mathrm{~N}_{2} \mathrm{O}_{4}$ (356.42): C, 67.34; H, 6.73; N, 7.86; O, 17.96. Found : C, 67.82; H, 6.37; N, 7.42; O, 17.55.

\section{5. conclusion}

A series of chitosan decorated copper nanoparticles were successfully synthesized using green methods. All the investigated catalysts showed high catalytic performance towards the synthesis, in acceptable yield, of novel quinoline derivatives at very short time under ultrasonic irradiation. $\mathrm{Cu}-\mathrm{CS}$ NPs catalyst showed superior TOF for the synthesis of novel quinoline derivatives of expected biological activity. Thanks to the well dispersion of nanoparticles over natural inactive good support the pronounced superior catalytic efficacy was attained. The re-use of this particular catalyst for five 
times without appreciable change in its catalytic efficacy open the gate towards promising noble metal free catalysts for fine chemical products utilizing green protocol.

\section{References}

[1] Larsen, R. D.; Corley, E. G.; King, A. O.; Carroll, J. D.; Davis, P.; Verhoeven, T. R.; Reider, P. J.; Labelle, M.; Gauthier, J. Y.; Xiang, Y. B., Practical route to a new class of LTD4 receptor antagonists. The Journal of Organic Chemistry 1996, 61 (10), 3398-3405.

[2] Kohn, L. K.; Pavam, C.; Veronese, D.; Coelho, F.; De Carvalho, J.; Almeida, W. P., Antiproliferative effect of Baylis-Hillman adducts and a new phthalide derivative on human tumor cell lines. European journal of medicinal chemistry 2006, 41 (6), 738-744.

[3] Caprio, V.; Guyen, B.; Opoku-Boahen, Y.; Mann, J.; Gowan, S. M.; Kelland, L. M.; Read, M. A.; Neidle, S., A novel inhibitor of human telomerase derived from $10 \mathrm{H}$-indolo [3, 2-b] quinoline. Bioorganic $\mathcal{E}$ medicinal chemistry letters 2000, 10 (18), 2063-2066.

[4] Roma, G.; Di Braccio, M.; Grossi, G.; Mattioli, F.; Ghia, M., 1, 8-Naphthyridines IV. 9-Substituted N, N-dialkyl-5-(alkylamino or cycloalkylamino)[1, 2, 4] triazolo [4, 3-a][1, 8] naphthyridine-6carboxamides, new compounds with anti-aggressive and potent anti-inflammatory activities. European journal of medicinal chemistry 2000, 35 (11), 1021-1035.

[5] Bekhit, A. A.; El-Sayed, O. A.; Aboulmagd, E.; Park, J. Y., Tetrazolo [1, 5-a] quinoline as a potential promising new scaffold for the synthesis of novel anti-inflammatory and antibacterial agents. European journal of medicinal chemistry 2004, 39 (3), 249-255.

[6] Dubé, D.; Blouin, M.; Brideau, C.; Chan, C.-C.; Desmarais, S.; Ethier, D.; Falgueyret, J.-P.; Friesen, R. W.; Girard, M.; Girard, Y., Quinolines as potent 5-lipoxygenase inhibitors: synthesis and biological profile of L-746,530. Bioorganic \& medicinal chemistry letters 1998, 8 (10), 1255-1260.

[7] Kalluraya, B.; Sreenivasa, S., Synthesis and pharmacological properties of some quinoline derivatives. Il Farmaco 1998, 53 (6), 399-404.

[8] Ferrarini, P. L.; Mori, C.; Badawneh, M.; Manera, C.; Martinelli, A.; Romagnoli, F.; Saccomanni, G.; Miceli, M., Unusual nitration of substituted 7-amino-1, 8-naphthyridine in the synthesis of compounds with antiplatelet activity. Journal of heterocyclic chemistry 1997, 34 (5), 1501-1510.

[9] Das, B.; Ravikanth, B.; Ramu, R.; Rao, B. V., An efficient one-pot synthesis of polyhydroquinolines at room temperature using HY-zeolite. Chemical and pharmaceutical bulletin 2006, 54 (7), 1044-1045.

[10] Sapkal, S. B.; Shelke, K. F.; Shingate, B. B.; Shingare, M. S., Nickel nanoparticle-catalyzed facile and efficient one-pot synthesis of polyhydroquinoline derivatives via Hantzsch condensation under solvent-free conditions. Tetrahedron Letters 2009, 50 (15), 1754-1756.

[11] Dondoni, A.; Massi, A.; Minghini, E.; Bertolasi, V., Multicomponent Hantzsch cyclocondensation as a route to highly functionalized 2-and 4-dihydropyridylalanines, 2-and 4-pyridylalanines, and their N-oxides: preparation via a polymer-assisted solution-phase approach. Tetrahedron 2004, 60 (10), 23112326. 
[12] Karade, N. N.; Budhewar, V. H.; Shinde, S. V.; Jadhav, W. N., L-proline as an efficient organocatalyst for the synthesis of polyhydroquinoline via multicomponent Hantzsch reaction. Letters in Organic Chemistry 2007, 4 (1), 16-19.

[13] Mason, T.; Sonochemistry, L. J., Ellis Horwood Ltd. Chichester, England 1988, 17-50.

[14] Hussain, S.; Jadhav, S.; Rai, M.; Farooqui, M., ONE-POT NEDA CATALYZED KNOVENAGEL CONDENSATION UNDER ULTRASONIC IRRADIATION IN SOLVENT-FREE MEDIUM. International Journal of Pharmaceutical, Chemical \& Biological Sciences 2014, 4 (1).

[15] Polshettiwar, V.; Varma, R. S., Green chemistry by nano-catalysis. Green Chemistry 2010, 12 (5), $743-$ 754.

[16] Rostamizadeh, S.; Shadjou, N.; Azad, M.; Jalali, N., ( $\alpha$-Fe2O3)-MCM-41 as a magnetically recoverable nanocatalyst for the synthesis of pyrazolo [4, 3-c] pyridines at room temperature. Catalysis Communications 2012, 26, 218-224.

[17] Mokhtar, M.; Saleh, T.; Ahmed, N.; Al-Thabaiti, S.; Al-Shareef, R., An eco-friendly N-sulfonylation of amines using stable and reusable $\mathrm{Zn}-\mathrm{Al}$-hydrotalcite solid base catalyst under ultrasound irradiation. Ultrasonics sonochemistry 2011, 18 (1), 172-176.

[18] Mokhtar, M.; Saleh, T. S.; Basahel, S. N., Mg-Al hydrotalcites as efficient catalysts for aza-Michael addition reaction: a green protocol. Journal of Molecular Catalysis A: Chemical 2012, 353, 122-131.

[19] Saleh, T. S.; Narasimharao, K.; Ahmed, N. S.; Basahel, S. N.; Al-Thabaiti, S. A.; Mokhtar, M., MgAl hydrotalcite as an efficient catalyst for microwave assisted regioselective 1, 3-dipolar cycloaddition of nitrilimines with the enaminone derivatives: A green protocol. Journal of Molecular Catalysis A: Chemical 2013, 367, 12-22.

[20] Narasimharao, K.; Al-Sabban, E.; Saleh, T. S.; Gallastegui, A. G.; Sanfiz, A. C.; Basahel, S.; AlThabaiti, S.; Alyoubi, A.; Obaid, A.; Mokhtar, M., Microwave assisted efficient protocol for the classic Ullmann homocoupling reaction using $\mathrm{Cu}-\mathrm{Mg}-\mathrm{Al}$ hydrotalcite catalysts. Journal of Molecular Catalysis A: Chemical 2013, 379, 152-162.

[21] Bagabas, A. A.; Mokhtar, M.; Akhmedov, V. M.; Narasimharao, K.; Basahel, S. N.; Al-Rabiah, A., $\mathrm{Ru}-\mathrm{C}-\mathrm{ZnO}$ Composite Catalysts for the Synthesis of Methyl Isobutyl Ketone via Single Step Gas Phase Acetone Self-Condensation. Catalysis letters 2014, 144 (7), 1278-1288.

[22] Basahel, S. N.; Ahmed, N. S.; Narasimharao, K.; Mokhtar, M., Simple and efficient protocol for synthesis of pyrido [1, 2-a] pyrimidin-4-one derivatives over solid heteropolyacid catalysts. RSC Advances 2016, 6 (15), 11921-11932.

[23] Puthiaraj, P.; Ahn, W.-S., Highly active palladium nanoparticles immobilized on NH2-MIL-125 as efficient and recyclable catalysts for Suzuki-Miyaura cross coupling reaction. Catalysis Communications 2015, 65, 91-95.

[24] Wang, X.; Hu, P.; Xue, F.; Wei, Y., Cellulose-supported N-heterocyclic carbene-palladium catalyst: Synthesis and its applications in the Suzuki cross-coupling reaction. Carbohydrate polymers 2014, 114, 476-483.

[25] Huang, Q. Z.; Zhuo, L. H.; Guo, Y. C., Heterogeneous degradation of chitosan with H2O2 catalysed by phosphotungstate. Carbohydrate polymers 2008, 72 (3), 500-505. 
[26] Baig, R. N.; Varma, R. S., Copper on chitosan: a recyclable heterogeneous catalyst for azide-alkyne cycloaddition reactions in water. Green Chemistry 2013, 15 (7), 1839-1843.

[27] Yang, B.; Mao, Z.; Zhu, X.; Wan, Y., Functionalised chitosan as a green, recyclable, supported catalyst for the copper-catalysed Ullmann $\mathrm{CN}$ coupling reaction in water. Catalysis Communications 2015, 60, 92-95.

[28] Baig, R. N.; Nadagouda, M. N.; Varma, R. S., Ruthenium on chitosan: a recyclable heterogeneous catalyst for aqueous hydration of nitriles to amides. Green Chemistry 2014, 16 (4), 2122-2127.

[29] Yan, K.; Chen, A., Selective hydrogenation of furfural and levulinic acid to biofuels on the ecofriendly Cu-Fe catalyst. Fuel 2014, 115, 101-108.

[30] Hardy, J. J.; Hubert, S.; Macquarrie, D. J.; Wilson, A. J., Chitosan-based heterogeneous catalysts for Suzuki and Heck reactions. Green Chemistry 2004, 6 (1), 53-56.

[31] Qiu, Y.; Ma, Z.; Hu, P., Environmentally benign magnetic chitosan/Fe $3 \mathrm{O} 4$ composites as reductant and stabilizer for anchoring Au NPs and their catalytic reduction of 4-nitrophenol. Journal of Materials Chemistry A 2014, 2 (33), 13471-13478.

[32] Zhou, J.; Dong, Z.; Yang, H.; Shi, Z.; Zhou, X.; Li, R., Pd immobilized on magnetic chitosan as a heterogeneous catalyst for acetalization and hydrogenation reactions. Applied Surface Science 2013, 279, 360-366.

[33] Frindy, S.; el Kadib, A.; Lahcini, M.; Primo, A.; García, H., Copper Nanoparticles Stabilized in a Porous Chitosan Aerogel as a Heterogeneous Catalyst for C- S Cross-coupling. ChemCatChem 2015, 7 (20), 3307-3315.

[34] Manikandan, A.; Sathiyabama, M., Green synthesis of copper-chitosan nanoparticles and study of its antibacterial activity. Journal of Nanomedicine \& Nanotechnology 2015, 6 (1), 1.

[35] Huang, H.; Yang, X., Synthesis of chitosan-stabilized gold nanoparticles in the absence/presence of tripolyphosphate. Biomacromolecules 2004, 5 (6), 2340-2346.

[36] Abdollahi-Alibeik, M.; Hoseinikhah, S. S., ClO 4-/Zr-MCM-41 nanoparticles prepared at mild conditions: a novel solid acid catalyst for the synthesis of polyhydroquinolines. Journal of the Iranian Chemical Society 2016, 13 (7), 1339-1347.

[37] Abdollahi-Alibeik, M.; Rezaeipoor-Anari, A., Fe3O4@ B-MCM-41: A new magnetically recoverable nanostructured catalyst for the synthesis of polyhydroquinolines. Journal of Magnetism and Magnetic Materials 2016, 398, 205-214.

[38] Kassaee, M.; Masrouri, H.; Movahedi, F., ZnO-nanoparticle-promoted synthesis of polyhydroquinoline derivatives via multicomponent Hantzsch reaction. Monatshefte für ChemieChemical Monthly 2010, 141 (3), 317-322.

[39] Sudheesh, N.; Sharma, S. K.; Shukla, R. S., Chitosan as an eco-friendly solid base catalyst for the solvent-free synthesis of jasminaldehyde. Journal of molecular catalysis A: Chemical 2010, 321 (1-2), 7782. 
[40] Kumar, S.; Dutta, P.; Koh, J., A physico-chemical and biological study of novel chitosanchloroquinoline derivative for biomedical applications. International journal of biological macromolecules 2011, 49 (3), 356-361.

[41]Roberts, G. A., Chitin chemistry. Macmillan International Higher Education: 1992. 